\title{
Mobility of a One-Dimensional Confined File of Water Molecules as a Function of File Length
}

\author{
Sapar M. Saparov, ${ }^{1}$ Jochen R. Pfeifer, ${ }^{2}$ Loay Al-Momani, ${ }^{2}$ Guillem Portella, ${ }^{3}$ \\ Bert L. de Groot, ${ }^{3}$ Ulrich Koert, ${ }^{2}$ and Peter Pohl ${ }^{1, *}$ \\ ${ }^{1}$ Institut für Biophysik, Johannes Kepler Universität Linz, Altenbergerstraße 69, A-4040 Linz, Austria \\ ${ }^{2}$ Hans-Meerwein-Straße, Fachbereich Chemie, Philipps-Universität Marburg, Hans-Meerwein-Straße, D-35043 Marburg, Germany \\ ${ }^{3}$ Max Planck Institute for Biophysical Chemistry, Am Fassberg 11, D-37077 Göttingen, Germany
}

(Received 7 October 2005; published 10 April 2006)

\begin{abstract}
Confinement of water by pore geometry to a one-dimensional file of molecules interacting with the pore alters the diffusion coefficient $D_{W}$. Here we report an exponential dependence of $D_{W}$ on the number of water positions in the pore. The result is based on measurements of single channel water permeabilities of structurally similar peptidic nanopores of different length. The inconsistency with predictions from continuum or kinetic models indicates that pore occupancy is reduced in single file transport. In longer pores (e.g., in aquaporins) the presence of charged residues increases $D_{W}$.
\end{abstract}

PACS numbers: 87.15.Vv, 82.80.Fk, 83.50.Ha, 87.16.Dg

Selectivity of membrane channels is, in part, achieved by size exclusion. In the limiting case, pores are so narrow that water molecules and ions cannot pass each other. They accommodate a one-dimensional file of molecules that due to interactions with the pore clearly differs in its physical characteristics from bulk water. Similarly, confined water can be found, e.g., in reactive centers or in the binding pocket of receptors. Molecular dynamics simulations have revealed a broad spectrum of water mobilities in confined pores. Depending on pore geometry, reduced water mobility has been reported [1], as well as immobilized water [2] or drastically enhanced water mobility $[3,4]$.

In the following, we will use the diffusion coefficient $D_{W}$ to characterize the mobility of water molecules. In a pore that contains $n$ water molecules, $D_{W}$ is $n$ times larger than the solvent diffusion coefficient of the enclosed water chain [5]. $D_{W}$ is assumed to be independent of channel length, $L$. Rather, $D_{W}$ reflects the influence of the protein channel on water mobility; i.e., it is the average diffusivity a water molecule would have if it were the only molecule in the pore. $D_{W}$ can be predicted from reaction rate theory, assuming that the pore consists of a series of binding sites, $N$ [6]. Experimentally, $D_{W}$ can be derived from the hydraulic single channel permeability coefficient, $p_{f}[7]$ :

$$
p_{f}=\frac{\nu_{w} D_{W} n}{L^{2}},
$$

where $\nu_{W}$ is the molar volume of a water molecule. Assuming that the water density in the pore is the same as in bulk, i.e., $n=N, L=2 N r$, and $\nu_{w}=3 / 4 \pi r^{3}, D_{W}$ can be expressed as

$$
D_{W}=\frac{3 N p_{f}}{\pi r},
$$

where $r$ is the channel radius.

Inconsistencies of this simple theory have been recognized long ago. According to Eq. (2), the $p_{f}$ values of single and double length nystatin channels are expected to differ by a factor of 2 . In contrast, permeabilities of $10 \times$ $10^{-14} \mathrm{~cm}^{3} / \mathrm{s}$ and $1.5 \times 10^{-14} \mathrm{~cm}^{3} / \mathrm{s}$, respectively, have been found [8]. An analysis of known water permeabilities of other narrow channels suggests that generally, shorter channels tend to have a higher $p_{f}$ [9]. The highest $p_{f}$ of $4.8 \times 10^{-12} \mathrm{~cm}^{3} \mathrm{~s}^{-1}$ was measured for the bacterial potassium channel from Streptomyces lividans (KcsA) [10]. Assuming that the resistance to water flow is caused mainly by the selectivity filter $(n=4, r=0.15 \mathrm{~nm})$, it translates into a $D_{W}$ of $1.2 \times 10^{-3} \mathrm{~cm}^{2} \mathrm{~s}^{-1}$. In contrast, the fourfold longer water channel protein (nodulin-26, $n=18.3$ ) offers a $p_{f}$ of $0.3 \times 10^{-14} \mathrm{~cm}^{3} \mathrm{~s}^{-1}$ [11], which corresponds to a $D_{W}$ of $3.7 \times 10^{-6} \mathrm{~cm}^{2} \mathrm{~s}^{-1}$. Thus, $D_{W}$ inside a cylindrical pore may change a thousandfold, while the pore geometry remains essentially unchanged.

Unfortunately, the comparison of structurally very different channels does not allow attributing the dramatic increase of $D_{W}$ by over 1 order of magnitude above bulk mobility solely to $L$. Other factors, e.g., channel hydrophobicity, may have contributed as well.

To investigate the impact of $L$ on $D_{W}$, we now have explored peptidic nanopores which differ only in $L$. The known $p_{f}$ of gramicidin-A (gA) is compared to $p_{f}$ of minigramicidin (MNg) [12] and midigramicidin ( $\mathrm{MDg}$ ) [13]. To conserve the head-to-head conformation of gA dimers [14] in the shorter peptides, we have linked the monomers by a succinyl group [Fig. 1(a)]. $\mathrm{MNg}$ is four amino acids shorter than MDg that, in turn, differs by four amino acids from the gA dimer [12,13]. For all peptidic nanopores used, $N$ was determined from atomistic molecular dynamics simulations as the average number of molecules interacting solely with other water molecules in the channel direction (Table I). MDg and MNg offered, respectively, just one or two water positions less than $\mathrm{gA}$ [Fig. 1(b)].

The electrical single channel conductivities, $g$, of $\mathrm{MNg}$ and MDg in our thin solvent free membranes were monitored (Fig. 2) as they are known to be a function of bilayer 
(a)

$$
\text { minigramicidin (R= TBDPS) }
$$

(b)

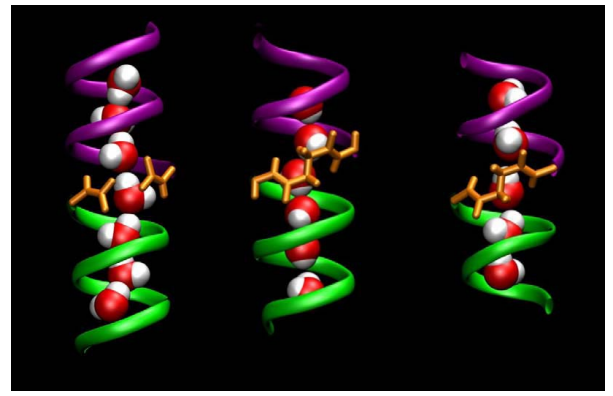

FIG. 1 (color online). (a) Structural formula of midigramici$\operatorname{din}(\mathrm{MDg})$ and minigramicidin $(\mathrm{MNg}) \quad(\mathrm{TBDPS}=$ tertButyldiphyenylsilyl). (b) Molecular dynamics simulations of gramicidin- $\mathrm{A}$ (gA), $\mathrm{MDg}$, and $\mathrm{MNg}$ (from left to right). As starting structures for the simulations the experimental structures with protein database entries 1MAG [27] and 1TKQ [13] were taken for $\mathrm{gA}$ and $\mathrm{MDg}$, respectively. In $\mathrm{MDg}$ the $N$ termini of gA and of a shorter peptide chain were covalently linked. The shorter chain resulted from the reduction of the pentadecapeptide sequence of $\mathrm{gA}$ to 11 residues. The structure of $\mathrm{MNg}$ was modeled by replication of the shorter chain of MDg. All molecular dynamics simulations were performed as described before [28] except that now we employed the optimized potentials for liquid simulations/all atoms [29] force field. Briefly, we used the GROMACS [30] software to simulate gA and derivatives embedded in an explicit Dimyristoylphosphatidylcholine membrane solvated in TIP4P water [31] at a constant temperature of $300 \mathrm{~K}$ and a pressure of $1 \mathrm{~atm}$. Particle-mesh Ewald [32] was used to compute long range electrostatics. Simulation times amounted to $100 \mathrm{~ns}$.

thickness [12,13]. For water flux measurements a large number, $m$, of channels was inserted into the bilayer. $m$ was assumed to be equal to the total membrane conductivity, $G$, divided by $g$. To determine $G$, a $1 \mathrm{kHz}$ square wave input voltage was applied to the membrane and the

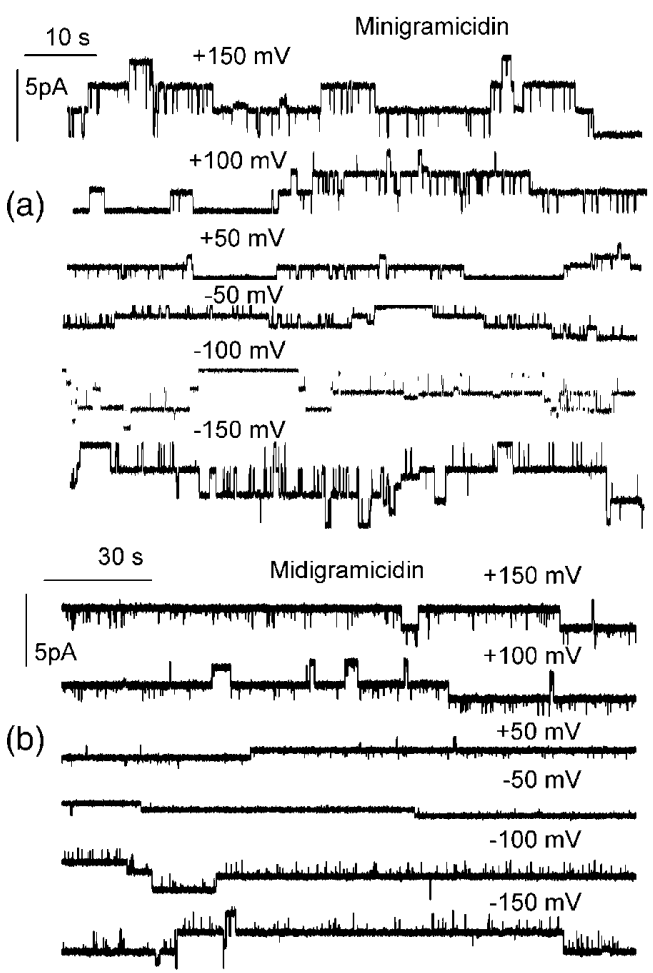

FIG. 2. Single channel recordings of (a) MNg and (b) MDg in lipid bilayers containing equal amounts of dimyristoylphosphatidylcholine (Sigma, Dreisenhofen, Germany) and $E$. coli lipid extract (Avanti Polar Lipids, Alabaster, AL). The bilayers were folded from monolayers in a $150 \mu \mathrm{m}$ diameter aperture of a polytetrafluoroethylene septum separating two aqueous phases [33]. The sampling frequency of the patch-clamp amplifier EPC-9 (HEKA Electronics, Germany) was fixed at $0.5 \mathrm{kHz}$ and a 4 pole Bessel with $3 \mathrm{~dB}$ corner frequency of $0.1 \mathrm{kHz}$ was chosen as the recording filter. The buffer $(p \mathrm{H} \mathrm{7.0)}$ contained (in mM) $10 \mathrm{MES}, 10 \mathrm{TRIS}, 0.2 \mathrm{CaCl}_{2}, 100 \mathrm{KCl}$.

amplified output signal was visualized by a digital oscilloscope.

Water flow though the peptide channels was measured by imposing an osmotic gradient across reconstituted planar bilayers and detecting the resulting small changes in ionic concentration close to the membrane surface $[15,16]$. With increasing peptide concentrations, more and more $\mathrm{Ca}^{2+}$ accumulated in the membrane vicinity, indicating an increase in water flow (Fig. 3). To derive $p_{f}$ the incremental water permeability induced by the peptidic channels, $P_{f, c}$ was plotted as a function of $G$ (Fig. 4):

TABLE I. The number of water positions, $N$, in the pores was derived from molecular dynamics simulations (compare Fig. 1). The osmotic single pore water permeability, $p_{f}$, was determined by scanning electrochemical microscopy (compare Figs. 3 and 4 ).

\begin{tabular}{lccc}
\hline \hline \multicolumn{1}{c}{ System } & Number of amino acids & Number of water positions & $p_{f}\left(10^{-14} \mathrm{~cm}^{3} \mathrm{~s}^{-1}\right)$ \\
\hline Gramicidin-A & 30 & 6.9 & 1.6 \\
Midigramicidin & 26 & 6.1 & 5.6 \\
Minigramicidin & 22 & 5.3 & 22 \\
\hline \hline
\end{tabular}




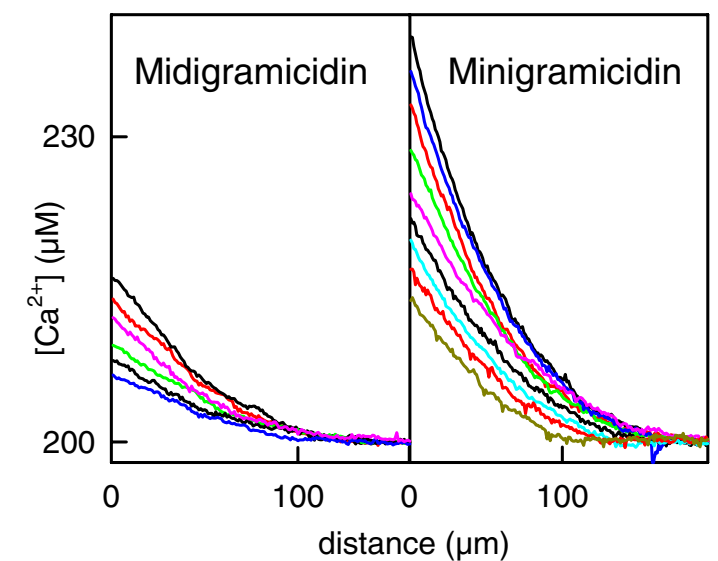

FIG. 3 (color online). Retention of $\mathrm{Ca}^{2+}$ in the membrane vicinity (hypoosmotic compartment) during steady state osmotic water flow induced by $1 \mathrm{M}$ urea. The changes in concentration were monitored as a function of the distance to the membrane by $\mathrm{Ca}^{2+}$ sensitive microelectrodes. Their tips (1-2 $\mu \mathrm{m}$ in diameter) were filled with Calcium Ionophore I Cocktail A (Fluka, Buchs, Switzerland). Stepwise movement of the electrodes relative to the membrane was realized by a hydraulic step drive (Narishige, Japan). The increase of $\mathrm{MNg}$ and $\mathrm{MDg}$ concentrations (from bottom to top) resulted in an increasing water flux and, thus, in an increasing concentration polarization. Except for $100 \mathrm{mM}$ cholinchloride and $1 \mathrm{mM} \mathrm{KCl}$ the buffer was as in Fig. 2.

$$
p_{f}=P_{f, c} A g / G
$$

where $A$ is the area of the membrane surface. For MDg and $\mathrm{MNg}$, the slopes of the current voltage dependence in $100 \mathrm{mM} \mathrm{KCl}$ indicated that $g$ was equal to 9.3 and $12.7 \mathrm{pS}$, respectively [Fig. 4(a)]. Correction of $g$ for $1 \mathrm{mM} \mathrm{KCl}$ [17] resulted in 0.2 and $0.27 \mathrm{pS}$, respectively. The respective $p_{f}$ were $2.2 \times 10^{-13} \mathrm{~cm}^{3} / \mathrm{s}$ and $0.56 \times$ $10^{-13} \mathrm{~cm}^{3} / \mathrm{s}$ as calculated from the slope of the regression lines [Fig. 4(b)]. Both values were 16-fold and fourfold higher than the $p_{f}$ of $1.6 \times 10^{-14} \mathrm{~cm}^{3} \mathrm{~s}^{-1}$ found for $\mathrm{gA}$ [15].

The huge increase in $p_{f}$ indicated that $D_{W}$ differed from pore to pore. Equation (2) allowed calculation of $D_{W}$. It depended exponentially on $N$ (Fig. 5). In the light of the classical continuous description [7] or kinetic formalisms [18] this result was counterintuitive since in gA, the contributions of all microscopic effects (like binding reactions) to $D_{W}$ were assumed to be minor in comparison to the access resistance [7,19].

If, however, "liquid-vapor" oscillations are hypothesized to occur, the rapid reduction of $D_{W}$ with $L$ emerges as a result of the partially hydrophobic character of the channel. Water transport occurs in bursts as shown by model channels $[3,4]$. This striking intermittency of water permeation changes dramatically with $r$ and/or $L$, leading to a strongly reduced rate of occupancy of the channel by water molecules [20].

Experimental evidence for a reduced occupancy was provided by the lack of proportionality between $p_{f}$ and

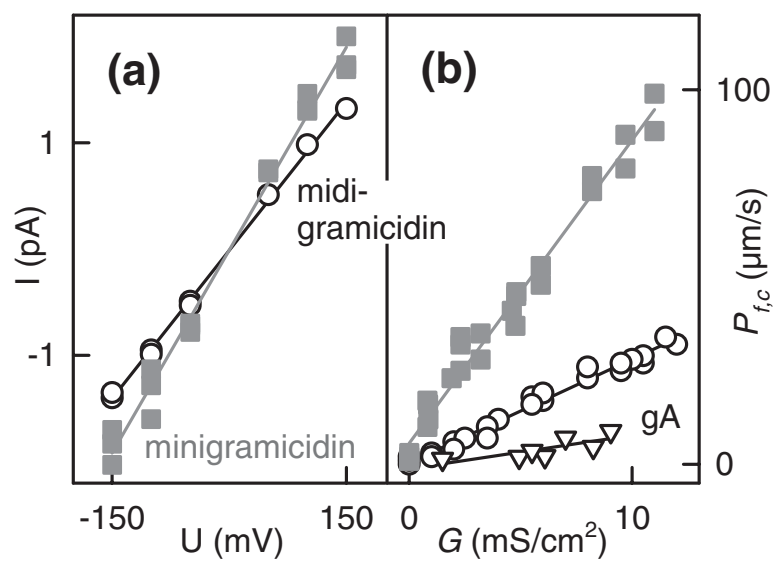

FIG. 4. Electrical (a) and hydraulic (b) single channel conductances. (a) The single channel currents were determined from the histograms of current traces and plotted against the applied voltage. Therefore, the TAC software (Bruxton Corporation, Seattle, WA) was used with a Gaussian filter of $37 \mathrm{~Hz}$. (b) Total membrane water permeability, $P_{f}$, as a function of integral electrical membrane conductivity, $G$. $P_{f}$ was calculated by fitting concentration profiles (Fig. 3) to $C(x)=C_{s} \exp \left(P_{f} C_{\mathrm{osm}} V_{w} x / D+\right.$ $\left.b x^{3} / 3 D\right)$, where $C_{s}, x, b, V_{w}$, and $D$ are, respectively, the solute concentration at the interface, the distance to the membrane, the stirring parameter, the molecular volume of water, and the bulk solute diffusion coefficient [34]. The transmembrane osmotic gradient, $C_{\text {osm }}$, has been obtained correcting the bulk urea concentration for dilution in the immediate membrane vicinity [35]. The data for $g A$ were taken from [15]. They were obtained in $1 \mathrm{mM} \mathrm{NaCl}$ (in $1 \mathrm{mM} \mathrm{KCl}$ the slope would have been even smaller due to the higher $\mathrm{K}^{+}$conductivity of $\mathrm{gA}$ ).

$1 / N$ [7] or $1 /(N+1)$ [18]. Both predictions resulted from the assumptions that the pores were constantly completely filled with water molecules $(N=n)$ [7] or that there was at most one vacancy in the pore $(n=N-1)$ [18]. At least for the very short MDg and MNg channels, both the classical continuous description [7] or the kinetic formalism [18] failed to predict the experimentally determined $p_{f}$.

A comparison with $p_{f}$ values of other single file channels with known $N$ indicates that the exponential dependence of $D_{W}$ on $N$ is a general feature (Fig. 5). Two groups of channels may be distinguished: (i) completely uncharged pores and (ii) pores with charged residues lining the wall. Both groups converge at the bacterial potassium channel KcsA, which directs 20 oxygen atoms toward the center of selectivity filter, and each oxygen atom is associated with a partial negative charge [21]. It is important to note that in all cases pure water flow is considered. The experimental conditions have been chosen such that almost all nanopores contained only water. The rare cases when an ion entered the pore may be neglected. This also holds for the aquaporins, which are generally impermeable to ions $[22,23]$ and also for KcsA that exhibits fast water flow only if there is no ion in the channel [10]. Thus, if intermittency of single file permeation is the reason for the reduced channel occupancy, this intermittency is not gated by 


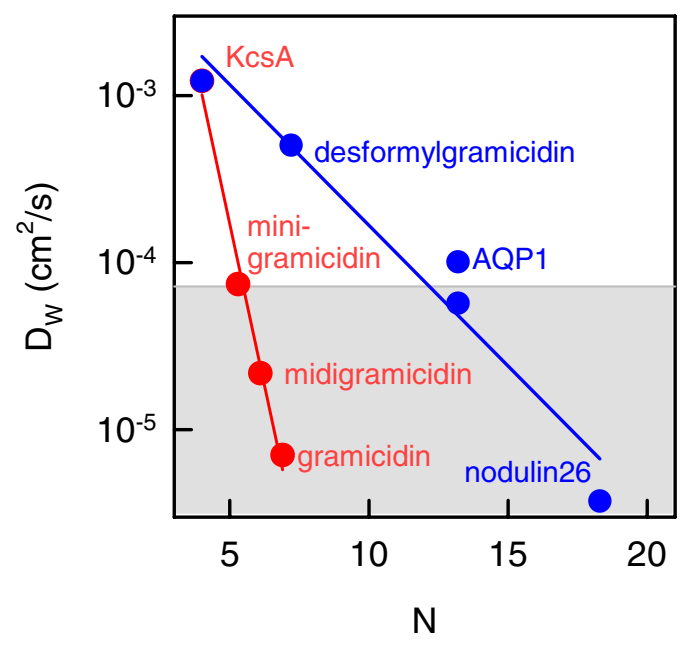

FIG. 5 (color online). Along with gA [15], MNg, MDg, desformylgramicdin [16], the human aquaporin-1 [36-38], the plant aquaporin nodulin 26 [11] and the bacterial potassium channel KcsA [10] are plotted. The gray area indicates $D_{W} \leqq$ water bulk mobility (for one-dimensional diffusion $7.2 \times 10^{-5} \mathrm{~cm}^{2} \mathrm{~s}^{-1}$ ).

ions. Herein, the single file transport differs from water permeation through wider pores that in silico was altered by the presence of an ion inside the channel [24,25]. Similarly, this intermittency cannot be mediated by electrostrictive behavior of water [26] because the requirement for a strong electric field contrasts (i) with the short circuited conditions we used for our water flow experiments and (ii) with the small size of the transmembrane gradient of the permeant ion (the polarization of $\mathrm{K}^{+}$is comparable the one of $\mathrm{Ca}^{2+}$ shown in Fig. 3).

In single file transport liquid-vapor oscillations have been visualized by simulations of carbon nanotubes only [3]. Whether they occur in proteinaceous channels as well remains to be shown. One of their major features, the reduced occupancy of the nanopores, has now been derived from the steep dependence of $p_{f}$ on $N$. If full occupancy is assumed or if the number of vacancies is limited to one, the effect of $N$ on $p_{f}$ has to be much weaker. Remarkably, the reduction of just two water positions resulted in an increase in water mobility of over 1 order of magnitude.

The project was supported by the Deutsche Forschungsgemeinschaft (SPP Micro- and Nanofluidics).

*Corresponding author.

Electronic address: peter.pohl@jku.at

[1] A. Berezhkovskii and G. Hummer, Phys. Rev. Lett. 89, 064503 (2002).

[2] R. J. Mashl, S. Joseph, N. R. Aluru, and E. Jakobsson, Nano Lett. 3, 589 (2003).

[3] G. Hummer, J.C. Rasaiah, and J.P. Noworyta, Nature (London) 414, 188 (2001).
[4] O. Beckstein and M. S. P. Sansom, Proc. Natl. Acad. Sci. U.S.A. 100, 7063 (2003).

[5] D. G. Levitt and G. Subramanian, Biochim. Biophys. Acta 373, 132 (1974).

[6] P. Lauger, Biochim. Biophys. Acta 311, 423 (1973).

[7] A. Finkelstein, Water Movement through Lipid Bilayers, Pores, and Plasma Membranes (Wiley \& Sons, New York, 1987), p. 1.

[8] R. Holz and A. Finkelstein, J. Gen. Physiol. 56, 125 (1970).

[9] P. Pohl, Biol. Chem. Hoppe-Seyler 385, 921 (2004).

[10] S. M. Saparov and P. Pohl, Proc. Natl. Acad. Sci. U.S.A. 101, 4805 (2004).

[11] R. L. Rivers et al., J. Biol. Chem. 272, 16256 (1997).

[12] H. D. Arndt, A. Knoll, and U. Koert, ChemBioChem. 2, 221 (2001).

[13] X. Xie et al., FEBS J. 272, 975 (2005).

[14] O. S. Andersen et al., Nat. Struct. Biol. 6, 609 (1999).

[15] P. Pohl and S. M. Saparov, Biophys. J. 78, 2426 (2000).

[16] S. M. Saparov, Y.N. Antonenko, R.E. Koeppe, and P. Pohl, Biophys. J. 79, 2526 (2000).

[17] E. Neher, J. Sandblom, and G. Eisenman, J. Membr. Biol. 40, 97 (1978).

[18] J. A. Hernandez and J. Fischbarg, J. Gen. Physiol. 99, 645 (1992).

[19] S. W. Chiu, S. Subramaniam, and E. Jakobsson, Biophys. J. 76, 1939 (1999).

[20] R. Allen, J. P. Hansen, and S. Melchionna, J. Chem. Phys. 119, 3905 (2003).

[21] Y. Zhou and R. MacKinnon, J. Mol. Biol. 333, 965 (2003).

[22] S. P. Tsunoda et al., J. Biol. Chem. 279, 11364 (2004).

[23] S. M. Saparov, S. P. Tsunoda, and P. Pohl, Biol. Cell 97, 545 (2005).

[24] R. Allen, S. Melchionna, and J. P. Hansen, Phys. Rev. Lett. 89, 175502 (2002).

[25] A. Anishkin and S. Sukharev, Biophys. J. 86, 2883 (2004).

[26] J. Dzubiella and J. P. Hansen, J. Chem. Phys. 122, 234706 (2005).

[27] R. R. Ketchem, B. Roux, and T. A. Cross, Structure 5, 1655 (1997).

[28] B. L. de Groot, D. P. Tieleman, P. Pohl, and H. Grubmuller, Biophys. J. 82, 2934 (2002).

[29] W. L. Jorgensen, D.S. Maxwell, and J. Tirado-Rives, J. Am. Chem. Soc. 118, 11225 (1996).

[30] D. Van der Spoel et al., J. Comput. Chem. 26, 1701 (2005).

[31] W. L. Jorgensen et al., J. Chem. Phys. 79, 926 (1983).

[32] T. Darden, D. York, and L. Pedersen, J. Chem. Phys. 98, 10089 (1993).

[33] M. Montal and P. Mueller, Proc. Natl. Acad. Sci. U.S.A. 69, 3561 (1972).

[34] P. Pohl, S. M. Saparov, and Y. N. Antonenko, Biophys. J. 72, 1711 (1997).

[35] P. Pohl, S. M. Saparov, and Y. N. Antonenko, Biophys. J. 75, 1403 (1998).

[36] J. C. Mathai et al., J. Biol. Chem. 271, 1309 (1996).

[37] M. L. Zeidel, S. V. Ambudkar, B. L. Smith, and P. Agre, Biochemistry 31, 7436 (1992).

[38] B. Yang and A.S. Verkman, J. Biol. Chem. 272, 16140 (1997). 